\title{
The Euler-Lagrange Equation for Interpolating Sequence of Landmark Datasets
}

\author{
Mirza Faisal Beg ${ }^{1}$, Michael Miller ${ }^{1}$, Alain Trouvé ${ }^{2}$, and Laurent Younes ${ }^{3}$ \\ 1 Center for Imaging Science \& Department of Biomedical Engineering, \\ The Johns Hopkins University, 301 Clark Hall, Baltimore, MD 21218 \\ \{mfbeg, mim\}@cis.jhu.edu http://www.cis.jhu.edu \\ 2 LAGA, Université Paris 13, France \\ trouve@zeus.math.univ-paris13.fr \\ 3 CMLA, Ecole Normale Supérieure de Cachan, \\ 61, avenue du President Wilson, F-94 235 \\ Cachan CEDEX, France younes@cmla.ens-cachan.fr \\ http://www.cmla.ens-cachan.fr/Utilisateurs/younes/
}

\begin{abstract}
Non-rigid registration of landmarked datasets is an important problem that finds many applications in medical image analysis. In this paper, we present a method for interpolating a sequence of landmarks. The sequence of landmarks may be a model of growth, where anatomical object boundaries are parametrized by landmarks and the growth processes generate a landmarked sequence in time. In a variational optimization framework, the matching diffeomorphism for this problem is generated from a gradient algorithm based on the EulerLagrange equation of a cost framed in the inexact matching setting.
\end{abstract}

\section{Introduction}

The problem of generating dense, non-rigid transformations for sparse landmark matching has been studied by many groups, in particular in the small deformation setting by Bookstein 3] and in the large deformation, diffeomorphism setting by Joshi [7], Younes [4]. In applications relating to analysis of structures in medical images, it is natural to study diffeomorphic transformations which are invertible and smooth functions. The one-to-one property of these transformations ensures that disjoint sets remain disjoint and no fusion of points occur. The continuity properties ensure that connected sets remain connected and neighbourhoods are preserved. Smoothness properties of these transformations preserve the smoothness properties of anatomical object boundaries. In the large deformation setting as proposed by Christensen [5] for the image matching case and extended to landmark matching by Joshi [7], such a transformation linking given landmark datasets is generated as the evolution of a flow of a smooth velocity vector-field, and constraints on smoothness of the vector-fields determine the existence and uniqueness of transformations in the space of diffeomorphisms [6]8]. 
An important extension to this problem is finding a diffeomorphism interpolating a sequence of landmarks, which may be modelling for example, the trajectory of a set of template landmarks in space and time. These indexed landmarked datasets may be derived from images taken of anatomical structures that are changing shape and form under the influence of growth or neurodegenerative processeses. The estimation of this interpolating diffeomorphism, in the framework of large deformations inexact matching is achieved by the variational optimization of a cost or energy functional containing a term that measures the smoothness of the estimated transformation, which is framed in the context of smoothness of vector fields that generate this transformation, and is maximized, while concurrently minimizing a term that measures the error in landmark positions after transformation. We present this inexact matching cost and derive its associated Euler-Lagrange equation in the space of smooth vector fields that generate this transformation. We specialize to the case where the matching is between a pair of landmark datasets instead of a sequence of landmark dataset and present a gradient algorithm based on the corresponding Euler-Lagrange equation to estimate the matching transformation.

\section{Mathematical Setup and Notation}

Let the background space $\Omega$ be a bounded domain with piecewise $C^{1}$ boundary on $\mathbb{R}^{n}$ on which the image functions $I: \Omega \rightarrow \mathbb{R}^{d}$ and the sparse image representations using landmarks $\mathbf{x}=\left\{x_{k} \in \Omega\right\}_{k=1, \ldots, N}$ are defined. A homeomorphism on the background space is a bijective (invertible) function $\varphi: \Omega \rightarrow \Omega$, which, with its inverse $\varphi^{-1}$ is continuous. Let the set of homeomorphisms acting on the background space be denoted by $\operatorname{Hom}(\Omega)$. The homeomorphisms form a group for the usual law of composition $\psi \cdot \varphi \doteq \psi \circ \varphi$. Moreover, for any $\varphi \in \operatorname{Hom}(\Omega)$ and any landmark set $\mathbf{x}, \varphi \cdot \mathbf{x} \doteq\left\{\varphi\left(x_{k}\right)\right\}_{k=1, \ldots, N}$ defines an action of $\operatorname{Hom}(\Omega)$ on the set of landmarks. Let $\mathcal{G}$ be a sub-group of $\operatorname{Hom}(\Omega)$ (for instance the set diffeomorphisms $\operatorname{Diff}(\Omega)$ where any $\varphi \in \operatorname{Diff}(\Omega)$ is, with its inverse, continuously differentiable).

Let $v:[0,1] \rightarrow V$ be a time-dependent velocity vector-field where $V$ is a Hilbert space of smooth, compactly supported vector fields on $\Omega$. Let such a velocity vector field define the evolution of a curve $\phi^{v}:[0,1] \rightarrow \mathcal{G}$ via the evolution equation

$$
\frac{d}{d t} \phi_{t}^{v}(x)=v_{t}\left(\phi_{t}^{v}(x)\right)
$$

where the subscript in $\phi^{v}$ is used to explicitly denote the dependence of $\phi$ on the associated velocity field $v$. The terminal point of the curve $\phi^{v}$ at $t=0$ is $\phi_{0}^{v}=I d \in \mathcal{G}$ where $I d$ is the identity transformation $I d(x)=x, \forall x \in \Omega$. The end point of the curve $\phi^{v}$ at time $t=1$ is the particular diffeomorphism $\phi_{1}^{v}=\varphi \in \mathcal{G}$ that links the given landmark datasets $\mathbf{x}$ and $\mathbf{y}$ such that $y_{k}=\varphi\left(x_{k}\right)$ and it is this element $\varphi$ that we compute as the end point $\phi_{1}^{v}$ associated to a flow $v$. Thus, we seek a time-dependant velocity vector field $v$ which when integrated 
via equation 1 generates the particular diffeomorphism matching the landmark datasets.

Existence and uniqueness properties of transformations generated via equation 1 depend on the smoothness constraints placed on vector fields allowed in $V$ 6[8]. One choice to ensure existence of diffeomorphisms as solutions to the ODE equation 1 has been to construct $V$ as the completion of $C_{c}^{\infty}\left(\Omega, \mathbb{R}^{n}\right)$ for the inner-product in space $V$ defined through a differential operator $L$ (denoting its adjoint as $\left.L^{\dagger}\right)$ on $C_{c}^{\infty}\left(\Omega, \mathbb{R}^{n}\right)$ given by:

$$
\langle f, g\rangle_{V} \doteq\langle L f, L g\rangle_{2}=\left\langle L^{\dagger} L f, g\right\rangle_{2},
$$

where $\langle,\rangle_{2}$ is the usual $L^{2}$-product for square integrable vector-fields on $\Omega$ and $f, g$ are vector-fields in $V$. It follows from the assumptions made in the construction of $V$ that it is a reproducing kernel Hilbert space, for each point $x \in \Omega$ and each tangent vector $\alpha_{x} \in T_{x} \Omega$, the linear form $\delta_{x}^{\alpha_{x}}: f \mapsto\left\langle f(x), \alpha_{x}\right\rangle_{\mathbb{R}^{n}}$ is continous for all $f \in V$. Then, it follows from the Riesz Representation theorem that there exists a representor $K_{x}^{\alpha_{x}} \in V$ of the linear form $\delta_{x}^{\alpha_{x}}$ such that

$$
\delta_{x}^{\alpha_{x}}(f)=\left\langle f(x), \alpha_{x}\right\rangle_{\mathbb{R}^{n}}=\left\langle K_{x}^{\alpha_{x}}, f\right\rangle_{V}
$$

and that representor is given by $K_{x}^{\alpha_{x}}(y)=k(x, y) \alpha_{x}$ for each point $y \in \Omega$. Since $\left\langle K_{x}^{\alpha_{x}}, f\right\rangle_{V}=\left\langle L^{\dagger} L K_{x}^{\alpha_{x}}, f\right\rangle_{2}$, we see by writing the $L^{2}$-product that $k(x, y)$ is the Green's function for the operator $L^{\dagger} L$. We will however not need to explicitly find the Green's function for a specified operator $L$, rather we can specify such a function that has the properties required of one, and following 4, we will choose $k(x, y)=\exp \left(-\|x-y\|^{2} / 2 \sigma^{2}\right) \mathrm{I}_{n \times n}$ where $\mathrm{I}_{n \times n}$ is an identity matrix. The gaussian kernel which is chosen does not conform with our usual assumption of null boundary conditions for the vector fields on $\Omega$. In the landmark case, using a more refined kernel would make the method more complex without really improving the quality.

Let the notation $\phi_{s, t}: \Omega \rightarrow \Omega$ denote the composition $\phi_{s, t}=\phi_{t} \circ\left(\phi_{s}\right)^{-1}$. The interpretation of $\phi_{s, t}(y)$ is that it is the position at time $t$ of a particle that is at position $y$ at time $s$. Therefore $\phi_{1}^{v}(x)=\phi_{0,1}^{v}(x)$ is the function that denotes the position at time $t=1$ of particle that is at position $x$ at time 0 . The variation of $\phi_{s, t}^{v}$ under the perturbation $v \mapsto v+\epsilon h$ is given by [1]:

$$
\partial_{h} \phi_{s, t}^{v}=\left(\frac{\partial}{\partial \epsilon} \phi_{s, t}^{v+\epsilon h}\right)_{\mid \epsilon=0}=\int_{s}^{t} D \phi_{u, t}^{v} \circ \phi_{s, u} h_{u} \circ \phi_{s, u} \mathrm{~d} u .
$$

\section{Estimation of Diffeomorphisms for Interpolating Space-Time Landmark Sequence}

Let $\mathbf{x}=\left\{x_{k}, k=1, \ldots, N\right\}$ at $t=0$ denote the template landmarks, and $\mathbf{y}(\mathbf{t})=$ $\left\{y_{k}(t), k=1, \ldots, N\right\}$ for $t \in(0,1]$ denote the time-indexed sequence of target landmarks in $\mathbb{R}^{n}$ characterizing for instance a sampling of the motion of template landmarks in space and time. The variational problem for the estimation of the element in the group of diffeomorphisms matching the time-indexed sequence of landmarks is given as follows. 
Theorem 1 (Space-Time Landmark Matching). Given template landmarks $\boldsymbol{x}=\left\{x_{k}, k=1, \ldots, N\right\}$ at $t=0$ and a sequence of time-indexed target landmarks $\left.\boldsymbol{y}(\boldsymbol{t})=\left\{y_{k}(t)\right\}, k=1, \ldots, N\right\}$ for $t \in(0,1]$. The velocity field $\hat{v} \in L^{2}([0,1], V)$ interpolating the landmarks is given by

$$
\hat{v}=\underset{v \in L^{2}([0,1], V)}{\operatorname{arginf}} E(v) \doteq \underbrace{\int_{0}^{1}\left\|v_{t}\right\|_{V}^{2} d t}_{E_{1}(v)}+\underbrace{\frac{1}{\sigma^{2}} \int_{0}^{1} \sum_{k=1}^{N}\left\|y_{k}(t)-\phi_{0, t}\left(x_{k}\right)\right\|_{\mathbb{R}^{n}}^{2} d t}_{E_{2}(v)}
$$

and satisfies the Euler-Lagrange equation given by

$$
\left(\nabla_{v} E\right)_{t}=2 v_{t}-\frac{2}{\sigma^{2}} \sum_{k=1}^{N} K_{x_{k}(t)}^{\left(\int_{t}^{1} D \phi_{t, u}^{v}\left(x_{k}(t)\right)^{t}\left(y_{k}(u)-x_{k}(u)\right) d u\right)}=0
$$

Proof. The $V$-gradient of the first term when $v \in L^{2}([0,1], V)$ is perturbed by $h \in L^{2}([0,1], V)$ is given by:

$$
\partial_{h} E_{1}(v)=2 \int_{0}^{1}\left\langle v_{t}, h_{t}\right\rangle_{V} d t
$$

The $V$-gradient of the second term in the cost (equation 5) is :

$$
\begin{aligned}
\partial_{h} E_{2}(v) & =\frac{-2}{\sigma^{2}} \int_{0}^{1} \sum_{k=1}^{N}\left\langle y_{k}(t)-\phi_{0, t}^{v}\left(x_{k}\right), \partial_{h} \phi_{0, t}^{v}\left(x_{k}\right)\right\rangle_{\mathbb{R}^{n}} d t \\
& \stackrel{(a)}{=} \frac{-2}{\sigma^{2}} \int_{0}^{1} \sum_{k=1}^{N}\langle y_{k}(t)-\phi_{0, t}^{v}\left(x_{k}\right), \underbrace{\left.\int_{0}^{t} D \phi_{u, t}^{v} \circ \phi_{0, u}^{v}\left(x_{k}\right) h_{u} \circ \phi_{0, u}^{v}\left(x_{k}\right) d u\right\rangle_{\mathbb{R}^{n}} d t}_{\partial_{h} \phi_{0, t}^{v}\left(x_{k}\right)} \\
& \stackrel{(b)}{=} \frac{-2}{\sigma^{2}} \int_{0}^{1} \int_{0}^{t} \sum_{k=1}^{N}\left\langle y_{k}(t)-\phi_{0, t}^{v}\left(x_{k}\right), D \phi_{u, t}^{v} \circ \phi_{0, u}^{v}\left(x_{k}\right) h_{u} \circ \phi_{0, u}^{v}\left(x_{k}\right)\right\rangle_{\mathbb{R}^{n}} d u d t \\
& \stackrel{(c)}{=} \frac{-2}{\sigma^{2}} \int_{0}^{1} \int_{u}^{1} \sum_{k=1}^{N}\left\langle y_{k}(t)-\phi_{0, t}^{v}\left(x_{k}\right), D \phi_{u, t}^{v} \circ \phi_{0, u}^{v}\left(x_{k}\right) h_{u} \circ \phi_{0, u}^{v}\left(x_{k}\right)\right\rangle_{\mathbb{R}^{n}} d t d u
\end{aligned}
$$

where (a) follows by writing the differential $\partial \phi_{0, t}^{v}\left(x_{k}\right)$, (b) follows from rearranging the integral and the inner-product, (c) follows by interchanging the order of integration with respect to $t$ and $u$. Interchanging the roles of $t$ and $u$ gives (d) to be: 


$$
\begin{aligned}
\partial_{h} E_{2}(v) & \stackrel{(d)}{=} \frac{-2}{\sigma^{2}} \int_{0}^{1} \int_{t}^{1} \sum_{k=1}^{N}\left\langle\left(y_{k}(u)-\phi_{0, u}^{v}\left(x_{k}\right)\right), D \phi_{t, u}^{v} \circ \phi_{0, t}^{v}\left(x_{k}\right) h_{t} \circ \phi_{0, t}^{v}\left(x_{k}\right)\right\rangle_{\mathbb{R}^{n}} d u d t \\
& \stackrel{(e)}{=} \frac{-2}{\sigma^{2}} \int_{0}^{1} \int_{t}^{1} \sum_{k=1}^{N}\left\langle D \phi_{t, u}^{v}\left(x_{k}(t)\right)^{t}\left(y_{k}(u)-x_{k}(u)\right), h_{t} \circ x_{k}(t)\right\rangle_{\mathbb{R}^{n}} d u d t \\
& \stackrel{(f)}{=}-\int_{0}^{1} \frac{2}{\sigma^{2}} \sum_{k=1}^{N}\left\langle\int_{t}^{1} D \phi_{t, u}^{v}\left(x_{k}(t)\right)^{t}\left(y_{k}(u)-x_{k}(u)\right) d u, h_{t} \circ x_{k}(t)\right\rangle_{\mathbb{R}^{n}} d t \\
& \stackrel{(g)}{=}-\int_{0}^{1}\left\langle\frac{2}{\sigma^{2}} \sum_{k=1}^{N} K_{x_{k}(t)}^{\left(\int_{t}^{1} D \phi_{t, u}^{v}\left(x_{k}(t)\right)^{t}\left(y_{k}(u)-x_{k}(u)\right) d u\right)}, h_{t}\right\rangle_{V} d t
\end{aligned}
$$

where (e) follows from writing $\phi_{0, t}^{v}\left(x_{k}\right)=x_{k}(t)$ and transposing the terms in the inner-product, (f) from rearranging the integral and the inner-product, (g) from using the Green's Kernel on $V$ transferring the inner-product from $\mathbb{R}^{n}$ to space $V$. Collecting the terms we get

$$
\partial_{h} E(v)=\int_{0}^{1}\left\langle 2 v_{t}-\frac{2}{\sigma^{2}} \sum_{k=1}^{N} K_{x_{k}(t)}^{\left(\int_{t}^{1} D \phi_{t, u}^{v}\left(x_{k}(t)\right)^{t}\left(y_{k}(u)-x_{k}(u)\right) d u\right)}, h_{t}\right\rangle
$$

and since the perturbation $h \in L^{2}([0,1], V)$ is arbitrary, we get equation 6 .

Corollary 1 (Static Landmark Matching). For target landmarks $\boldsymbol{y}$ at only one time-instant $t=1$ i. e. $\boldsymbol{y}=y(1)=\left\{y_{k}\right\}$, the Euler-Lagrange equation becomes:

$$
\left(\nabla_{v} E\right)_{t}=2 v_{t}-\frac{2}{\sigma^{2}} \sum_{k=1}^{N} K_{x_{k}(t)}^{\left(D \phi_{t, 1}^{v}\left(x_{k}(t)\right)^{t}\left(y_{k}-x_{k}(1)\right)\right)}=0
$$

\subsection{Brief Implementation Note}

We have implemented equation 9] and are currently working on implementing equation 6. We provide here a few remarks on the implementation, the details are given in [2. The calculation for the optimal velocity vector field for landmark matching proceeds via a gradient algorithm based on the Euler-Lagrange equation 9 The flow interval $[0, T]$, where $T=1$ by default but can be an positive real number, is discretized into $N$ intervals such that $N \delta=T, \delta$ being the size of each timestep. The simulations begin with simulation-step 0 where the estimate of velocity vector field is set to zero i. e. $v_{t_{j}}=0, \forall j \in[0, N-1]$. The gradient at simulation step $n$ is used to update the estimate of $v$ for simulation $n+1$ as

$$
v_{t}^{n+1}=v_{t}^{n}-\epsilon \nabla_{v^{n}} E_{t}^{n} .
$$

The discretized gradient at simulation step $n$ for each discretized time index along the flow $j \in[0, N-1]$ is given by:

$$
\nabla_{v^{n}} E_{t_{j}}^{n}=2 v_{t_{j}}^{n}-\frac{2}{\sigma^{2}} \sum_{k=1}^{N} K_{x_{k}\left(t_{j}\right)}^{\left(D \phi_{t_{j}, 1}^{v}\left(x_{k}^{v}\left(t_{j}\right)\right)^{t}\left(y_{k}-x_{k}^{v}(1)\right)\right)}
$$


Computation of the gradient requires the calculation of the Jacobian matrices $D \phi_{t, 1}^{v}\left(x_{k}(t)\right)^{t}$ which can be done by backward integration of the flow as these satisfy

$$
\frac{d}{d t} D \phi_{t, 1}^{v}\left(x_{k}(t)\right)=-D \phi_{t, 1}^{v}\left(x_{k}(t)\right) D v_{t}\left(x_{k}(t)\right)
$$

with starting at $t=1$ where $D \phi_{1,1}^{v}\left(x_{k}(1)\right)=I_{n \times n}$. Given the velocity field $v$, this is an ordinary differential equation in $D \phi_{t, 1}^{v}$ which can be solved using standard methods for reverse time integration.

\section{Results}

In the experiments presented, the Green's Kernel $k(x, y)=\exp \left(-\|x-y\|^{2} / 2 \sigma^{2}\right)$ was used with $\sigma=0.1$. This parameter controls the smoothing of the computed transformation, where larger values of $\sigma$ give transformations of greater smoothness. The confidence or error in landmark placement is modelled by the landmark placement noise standard deviation $\sigma$ in the second term of the energy $\frac{1}{\sigma^{2}} \sum_{k=1}^{N}\left\|y_{k}-\phi_{0,1}\left(x_{k}\right)\right\|_{\mathbb{R}^{n}}^{2}$ and two values of this parameter $\sigma=0.5$ and 1.0 were used. Smaller values of this parameter put a higher penalty to the mismatch of the final position of template landmarks from the corresponding target landmarks and therefore tend towards making the matching exact. Flow was discretized into 20 intervals of size 0.1 each.

The first experiment estimates the diffeomorphism matching the template landmarks at points $(16,26)$ and $(48,36)$ to the corresponding target landmarks at points $(48,26)$ and $(16,36)$ in a grid of size 64 by 64 pixels. In the second experiment, 30 landmarks each from two macaque cortex cryosection images of size 80 by 80 pixels were used.

The determinant of the Jacobian of the estimated mapping for the two values of landmark placement noise standard deviation are summarized in the table below.

\begin{tabular}{|c|c|c|}
\hline \multirow{3}{*}{ Experiment(Grid Size) } & \multicolumn{2}{|c|}{ Det. of Jacobian } \\
\hline & $\sigma=0.5$ & $\sigma=1.0$ \\
\hline & \multicolumn{2}{|c|}{$\min \max \min \max$} \\
\hline & \multicolumn{2}{|c|}{$\begin{array}{llll}0.1 & 7.35 & 0.13 & 6.34\end{array}$} \\
\hline Expt. 2(80 by 80$)$ & \multicolumn{2}{|c|}{$\begin{array}{llll}0.33 & 2.4 & 0.54 & 1.77\end{array}$} \\
\hline
\end{tabular}

Visual inspection shows the estimate mappings are smooth and even though the deformtion in the first experiment is severe, the transformation estimated is one to one. Execution times of the algorithm for both of these experiments was of the order of a few seconds.

\section{Discussion and Conclusion}

The main contribution of this work is two-fold. First, we present the EulerLagrange equation to interpolate between a sequence of landmarked datasets. 

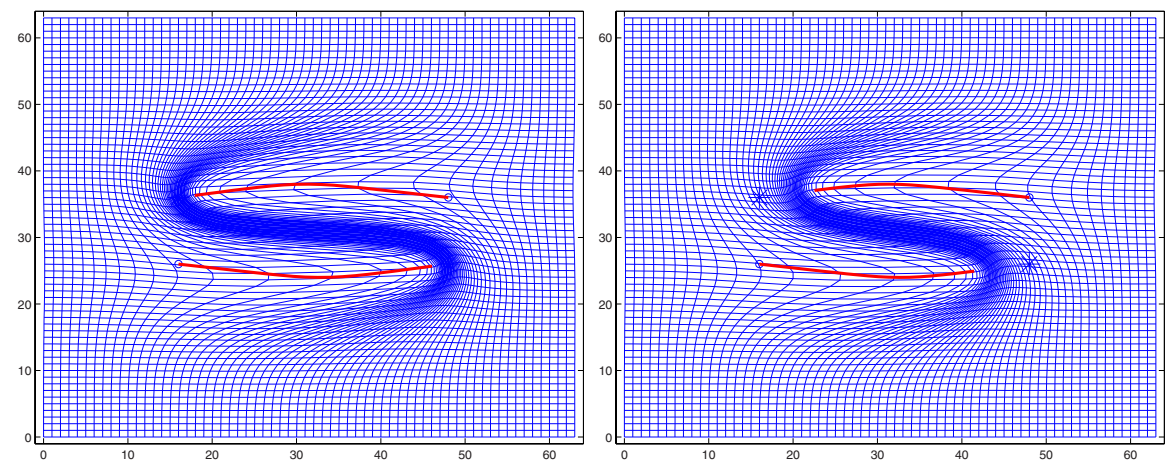

$\sigma=0.5$
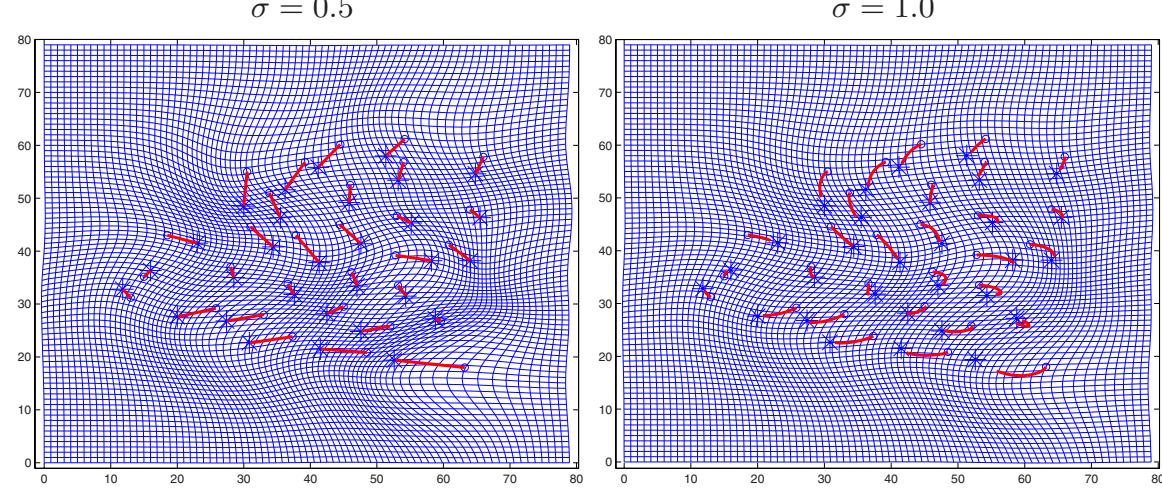

$\sigma=0.5$

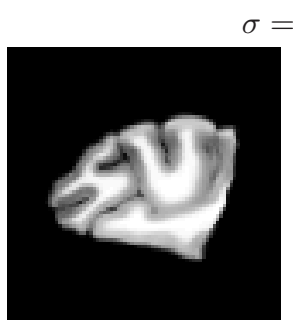

$\sigma=1.0$

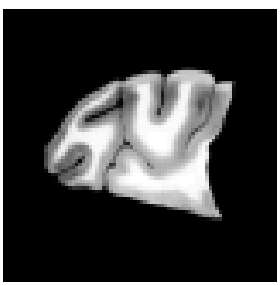

$I_{0}$

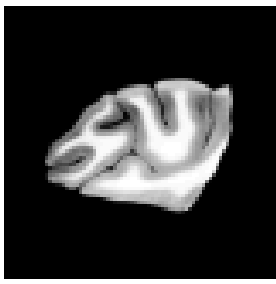

$I_{1}$

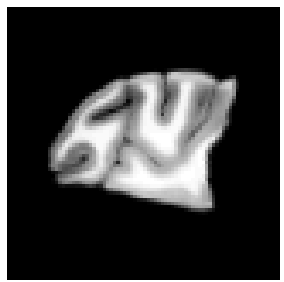

$I_{1}(\varphi)$

Fig. 1. The top row shows the diffeomorphism calculated for matching a simple test landmark dataset defined on domain of size 64 by 64 pixels. The bottom row shows the matching of two landmark datasets derived from placing landmarks on two macaque brain cryosection images of size 80 by 80 pixels. The circles are the template landmarks, the stars are the target landmarks and the line emanating from the template landmark is its trajectory to reach the corresponding target landmark. The matching experiments were run for two values of landmark placement noise $\sigma=0.5$ and $\sigma=1.0$. The bottom row shows the the Macaque cortex images $I_{0}$ and $I_{1}$ from which the landmarks in middle row were obtained, and also shows these images transformed using the estimated diffeomorphism the $\sigma=1$ case. 
Second, as a special case, we get a new method for computing a dense diffeomorphic transformation for interpolating between a pair of landmark datasets. The Euler-Lagrange equations are satisfied by the variational optimizer of the inexact matching cost in space $V$ of smooth vector fields. The gradient algorithm obtained from the Euler-Lagrange equation is thus in the space of smooth vector fields and therefore numerically stable and has nice convergence properties.

We present a challenging example of generating a diffeomorphic transformation for landmarks trajectories that criss-cross demonstrating the power of the large deformations setting. As the landmark placement noise variance decreases, the error in landmark matching decreases and this occurs at the expense of the smoothness of the estimated mapping, which decreases correspondingly. We also present the matching of landmark datasets derived from two Macaque cortex images. The estimated transformation is used for the non-rigid registration of these images where correspondance information is specified only via the sparse landmarked image representation.

\section{References}

1. M. F. Beg, M. Miller, A. Trouvé, and L. Younes. Computing metrics via geodesics on flows of diffeomorphisms. Accepted in the Internation Journal of Computer Vision, 2003.

2. Mirza Faisal Beg. Variational and Computational Methods for Flows of Diffeomorphisms in Image Matching and Growth in Computational Anatomy. PhD thesis, The Johns Hopkins University, July 2003.

3. F.L. Bookstein. Morphometric Tools for Landmark Data. Cambridge University Press, New York, 1991.

4. V. Camion and L. Younes. Geodesic interpolating splines. EMMCVPR, pages 513527, 2001.

5. G. E. Christensen, R. D. Rabbitt, and M. I. Miller. Deformable templates using large deformation kinematics. IEEE Transactions on Image Processing, 5(10):1435-1447, October 1996.

6. P. Dupuis, U. Grenander, and M.I. Miller. Variational problems on flows of diffeomorphisms for image matching. Quarterly of Applied Mathematics, LVI:587-600, September 1998.

7. S. Joshi and M. I. Miller. Landmark matching via large deformation diffeomorphisms. IEEE Transactions on Image Processing, 9(8):1357-1370, August 2000.

8. A. Trouvé. An infinite dimensional group approach for physics based models in patterns recognition. Preprint, 1995. 